\title{
Schur convexity for the ratios of the Hamy and generalized Hamy symmetric functions
}

Wei-Mao Qian

Correspondence: qwm661977@126. com

Huzhou Broadcast and TV

University, Huzhou 313000, China

\section{Abstract}

In this paper, we present the Schur convexity and monotonicity properties for the ratios of the Hamy and generalized Hamy symmetric functions and establish some analytic inequalities. The achieved results is inspired by the paper of Hara et al. [J. Inequal. Appl. 2, 387-395, (1998)], and the methods from Guan [Math. Inequal. Appl. 9, 797-805, (2006)]. The inequalities we obtained improve the existing corresponding results and, in some sense, are optimal.

2010 Mathematics Subject Classification: Primary 05E05; Secondary 26D20.

Keywords: Hamy symmetric function, generalized Hamy symmetric function, Schur convex, Schur concave

\section{Introduction}

Throughout this paper, we denote $\mathbb{R}_{+}^{n}=\left\{x=\left(x_{1}, x_{2}, \ldots, x_{n}\right) \mid x_{i}>0, i=1,2, \ldots, n\right\}$. For $x \in \mathbb{R}_{+}^{n}$, the Hamy symmetric function [1] is defined as

$$
F_{n}(x, r)=F_{n}\left(x_{1}, x_{2}, \ldots, x_{n} ; r\right)=\sum_{1 \leq i_{1}<i_{2}<\cdots<i_{r} \leq n}\left(\prod_{j=1}^{r} x_{i_{j}}\right)^{\frac{1}{r}},
$$

where $r$ is an integer and $1 \leq r \leq n$.

The generalized Hamy symmetric function was introduced by Guan [2] as follows

$$
F_{n}^{*}(x, r)=F_{n}^{*}\left(x_{1}, x_{2}, \ldots, x_{n} ; r\right)=\sum_{i_{1}+i_{2}+\cdots+i_{n}=r}\left(x_{1}^{i_{1}} x_{2}^{i_{2}} \ldots x_{n}^{i_{n}}\right)^{\frac{1}{r}},
$$

where $r$ is a positive integer.

In [2], Guan proved that both $F_{n}(x, r)$ and $F_{n}^{*}(x, r)$ are Schur concave in $\mathbb{R}_{+}^{n}$. The main of this paper is to investigate the Schur convexity for the functions $\frac{F_{n}(x, r)}{F_{n}(x, r-1)}$ and $\frac{F_{n}^{*}(x, r)}{F_{n}^{*}(x, r-1)}$ and establish some analytic inequalities by use of the theory of majorization.

For convenience of readers, we recall some definitions as follows, which can be found in many references, such as [3].

(c) 2011 Qian; licensee Springer. This is an Open Access article distributed under the terms of the Creative Commons Attribution License (http://creativecommons.org/licenses/by/2.0), which permits unrestricted use, distribution, and reproduction in any medium, provided the original work is properly cited. 
Definition 1.1. The $n$-tuple $x$ is said to be majorized by the $n$-tuple $y$ (in symbols $x \prec y$ ), if

$$
\sum_{i=1}^{k} x_{[i]} \leq \sum_{i=1}^{k} y_{[i]}, \quad \sum_{i=1}^{n} x_{[i]}=\sum_{i=1}^{n} y_{[i]},
$$

where $1 \leq k \leq n-1$, and $x_{[i]}$ denotes the $i$ th largest component of $x$.

Definition 1.2. Let $E \subseteq \mathbb{R}^{n}$ be a set. A real-valued function $F: E \rightarrow \mathbb{R}$ is said to be Schur convex on $E$ if $F(x) \leq F(y)$ for each pair of $n$-tuples $x=\left(x_{1}, \ldots, x_{n}\right)$ and $y=\left(y_{1}, \ldots\right.$, $y_{n}$ ) in $E$, such that $x \prec y$. $F$ is said to be Schur concave if $-F$ is Schur convex.

The theory of Schur convexity is one of the most important theories in the fields of inequalities. It can be used in combinatorial optimization [4], isoperimetric problems for polytopes [5], theory of statistical experiments [6], graphs and matrices [7], gamma functions [8], reliability and availability [9], optimal designs [10] and other related fields.

Our aim in what follows is to prove the following results.

Theorem 1.1. Let $x \in \mathbb{R}_{+}^{n}, 2 \leq r \leq n$ is an integer, then the function $\phi_{r}(x)=\frac{F_{n}(x, r)}{F_{n}(x, r-1)}$ is Schur concave in $\mathbb{R}_{+}^{n}$ and increasing with respect to $x_{i}(i=1,2, \ldots$, $n)$.

Theorem 1.2. Let $x \in \mathbb{R}_{+}^{n}, 2 \leq r \leq n$ is an integer, then the function $\phi_{r}^{*}(x)=\frac{F_{n}^{*}(x, r)}{F_{n}^{*}(x, r-1)}$ is Schur concave in $\mathbb{R}_{+}^{n}$ and increasing with respect to $x_{i}(i=1,2, \ldots n)$.

Corollary 1.1. If $x_{i}>0, i=1,2, \ldots, n, \sum_{i=1}^{n} x_{i}=s$ and that $c \geq s$, then

$$
\frac{G_{n}(x)}{G_{n}(c-x)}=\frac{F_{n}(x, n)}{F_{n}(c-x, n)} \leq \frac{F_{n}(x, n-1)}{F_{n}(c-x, n-1)} \leq \cdots \leq \frac{F_{n}(x, 1)}{F_{n}(c-x, 1)}=\frac{A_{n}(x)}{A_{n}(c-x)}
$$

and

$$
\frac{G_{n}(x)}{G_{n}(c+x)}=\frac{F_{n}(x, n)}{F_{n}(c+x, n)} \leq \frac{F_{n}(x, n-1)}{F_{n}(c+x, n-1)} \leq \cdots \leq \frac{F_{n}(x, 1)}{F_{n}(c+x, 1)}=\frac{A_{n}(x)}{A_{n}(c+x)},
$$

where $A_{n}(x)=\frac{1}{n} \sum_{i=1}^{n} x_{i}, G_{n}(x)=\left(\prod_{i=1}^{n} x_{i}\right)^{\frac{1}{n}}$ are the arithmetic and geo-metric means of $x$, respectively.

Corollary 1.2. If $x_{i}>0, i=1,2, \ldots, n, \sum_{i=1}^{n} x_{i}=s$ and that $c \geq s$, then

$$
\frac{F_{n}^{*}(x, r)}{F_{n}^{*}(c-x, r)} \leq \frac{F_{n}^{*}(x, r-1)}{F_{n}^{*}(c-x, r-1)} \leq \cdots \leq \frac{F_{n}^{*}(x, 2)}{F_{n}^{*}(c-x, 2)} \leq \frac{F_{n}^{*}(x, 1)}{F_{n}^{*}(c-x, 1)}=\frac{A_{n}(x)}{A_{n}(c-x)}
$$

and

$$
\frac{F_{n}^{*}(x, r)}{F_{n}^{*}(c+x, r)} \leq \frac{F_{n}^{*}(x, r-1)}{F_{n}^{*}(c+x, r-1)} \leq \cdots \leq \frac{F_{n}^{*}(x, 2)}{F_{n}^{*}(c+x, 2)} \leq \frac{F_{n}^{*}(x, 1)}{F_{n}^{*}(c+x, 1)}=\frac{A_{n}(x)}{A_{n}(c+x)}
$$

\section{Lemmas}

In order to establish our main results, we need several lemmas, which we present in this section. 
Lemma 2.1 (see [3]). Let $E \subseteq \mathbb{R}^{n}$ be a symmetric convex set with nonempty interior $\operatorname{int} E$ and $\phi: E \rightarrow \mathbb{R}$ be a continuous symmetric function. If $\phi$ is differentiable on int $E$, then $\phi$ is Schur convex (or Schur concave, respectively) on $E$ if and only if

$$
\left(x_{i}-x_{j}\right)\left(\frac{\partial \varphi}{\partial x_{i}}-\frac{\partial \varphi}{\partial x_{j}}\right) \geq 0 \quad \text { (or } \leq 0 \text {, respectively) }
$$

for all $i, j=1,2, \ldots, n$ and $x=\left(x_{1}, \ldots, x_{n}\right) \in \operatorname{int} E$.

The $r$ th elementary symmetric function (see [11]) is defined as

$$
E_{n}(x, r)=E_{n}\left(x_{1}, x_{2}, \ldots, x_{n} ; r\right)=\sum_{1 \leq i_{1}<i_{2}<\cdots<i_{r} \leq n}\left(\prod_{j=1}^{r} x_{i_{j}}\right),
$$

where $1 \leq r \leq n$ is a positive integer, and $E_{n}(x, 0)=1$.

By (2.1) and simple computations, we have the following lemma.

Lemma 2.2. Let $x \in \mathbb{R}_{+}^{n}, 1 \leq i \leq n$, if

$$
\overline{x_{i}}=\left(x_{1}, x_{2}, \ldots, x_{i-1}, x_{i+1}, \ldots, x_{n}\right) .
$$

Then,

$$
E_{n}\left(x_{1}, x_{2}, \ldots, x_{n} ; r\right)=x_{i} E_{n-1}\left(\overline{x_{i}}, r-1\right)+E_{n-1}\left(\overline{x_{i}}, r\right) .
$$

Lemma 2.3 (see [11]). Let $x \in \mathbb{R}_{+}^{n} r$ is an integer and $1 \leq r \leq n-1$.

Then,

$$
\left(E_{n}(x, r)\right)^{2}>E_{n}(x, r-1) E_{n}(x, r+1) .
$$

Another important symmetric function is the complete symmetric function (see [3]), which is defined by

$$
C_{r}(x)=C_{r}\left(x_{1}, x_{2}, \ldots, x_{n}\right)=\sum_{i_{1}+i_{2}+\cdots+i_{n}=r} x_{1}^{i_{1}} x_{2}^{i_{2}} \ldots x_{n}^{i_{n}}
$$

where $i_{1}, i_{2}, \ldots, i_{n}$ are non-negative integer, $r \in\{1,2, \ldots\}$ and $C_{0}(x)=1$.

Lemma 2.4 (see [12]). Let $x_{i}>0, i=1,2, \ldots, n$, and $\overline{x_{i}}=\left(x_{1}, x_{2}, \ldots, x_{i-1}, x_{i+1}, \ldots, x_{n}\right)$. Then,

$$
C_{r}(x)=x_{i} C_{r-1}(x)+C_{r}\left(\overline{x_{i}}\right) .
$$

Lemma 2.5 (see [13]). If $0<r<s, x \in \mathbb{R}_{++}^{n}$, then

$$
C_{r}(x) C_{s-1}(x)>C_{r-1} C_{s}(x) .
$$

Lemma 2.6 (see [14]). If $x_{i}>0, i=1,2, \ldots, n, \sum_{i=1}^{n} x_{i}=s$ and $c \geq s$, then

$$
\begin{aligned}
& \text { (1) } \frac{c-x}{\frac{n c}{s}-1}=\left(\frac{c-x_{1}}{\frac{n c}{s}-1}, \frac{c-x_{2}}{\frac{n c}{s}-1}, \ldots, \frac{c-x_{n}}{\frac{n c}{s}-1}\right) \prec\left(x_{1}, x_{2}, \ldots, x_{n}\right)=x_{\prime \prime} \\
& \text { (2) } \frac{c+x}{s+n c}=\left(\frac{c+x_{1}}{s+n c}, \frac{c+x_{2}}{s+n c}, \ldots, \frac{c+x_{n}}{s+n c}\right) \prec\left(\frac{x_{1}}{s}, \frac{x_{2}}{s}, \ldots, \frac{x_{n}}{s}\right)=\frac{x}{s} .
\end{aligned}
$$

\section{Proof of Theorems}

Proof of Theorem 1.1. It is obvious that $\varphi_{r}(x)$ is symmetric and has continuous partial derivatives in $\mathbb{R}_{+}^{n}$. By Lemma 2.1, we only need to prove that 


$$
\left(x_{1}-x_{2}\right)\left(\frac{\partial \phi_{r}(x)}{\partial x_{1}}-\frac{\partial \phi_{r}(x)}{\partial x_{2}}\right) \leq 0 .
$$

For any fixed $2 \leq r \leq n$, let $u_{i}=\sqrt[r]{x_{i}}, i=1,2, \ldots, n$ and $u=\left(u_{1}, u_{2}, \ldots, u_{n}\right) \in \mathbb{R}_{+}^{n}$, we have

$$
\phi_{r}(x)=\frac{F_{n}(x, r)}{F_{n}(x, r-1)}=\frac{E_{n}(u, r)}{E_{n}(u, r-1)} .
$$

Differentiating $\varphi_{r}(x)$ with respect to $x_{1}$ yields

$$
\frac{\partial \phi_{r}(x)}{\partial x_{1}}=\frac{1}{E_{n}^{2}(u, r-1)}\left[E_{n}(u, r-1) \frac{\partial E_{n}(u, r)}{\partial u_{1}} \frac{\partial u_{1}}{\partial x_{1}}-E_{n}(u, r) \frac{\partial E_{n}(u, r-1)}{\partial u_{1}} \frac{\partial u_{1}}{\partial x_{1}}\right] .
$$

Using Lemma 2.2 repeatedly, we get

$$
\begin{gathered}
E_{n}(u, r)=u_{1} u_{2} E_{n-2}\left(u_{3}, \ldots, u_{n} ; r-2\right)+\left(u_{1}+u_{2}\right) E_{n-2}\left(u_{3}, \ldots, u_{n} ; r-1\right) \\
+E_{n-2}\left(u_{3}, \ldots, u_{n} ; r\right) .
\end{gathered}
$$

Equations (3.2) and (3.3) lead to

$$
\frac{\partial \phi_{r}(x)}{\partial x_{1}}=\frac{1}{r E_{n}^{2}(u, r-1)}\left(u_{1}^{1-r} u_{2} A+u_{1}^{1-r} B\right),
$$

where

$$
A=E_{n}(u, r-1) E_{n-2}\left(u_{3}, \ldots, u_{n} ; r-2\right)-E_{n}(u, r) E_{n-2}\left(u_{3}, \ldots, u_{n} ; r-3\right)
$$

and

$$
B=E_{n}(u, r-1) E_{n-2}\left(u_{3}, \ldots, u_{n} ; r-1\right)-E_{n}(u, r) E_{n-2}\left(u_{3}, \ldots, u_{n} ; r-2\right) .
$$

Similarly, we can deduce that

$$
\frac{\partial \phi_{r}(x)}{\partial x_{2}}=\frac{1}{r E_{n}^{2}(u, r-1)}\left(u_{1} u_{2}^{1-r} A+u_{2}^{1-r} B\right) .
$$

From (3.4) and (3.5), one has

$$
\begin{aligned}
& \left(x_{1}-x_{2}\right)\left(\frac{\partial \phi_{r}(x)}{\partial x_{1}}-\frac{\partial \phi_{r}(x)}{\partial x_{2}}\right) \\
& =\frac{x_{1}-x_{2}}{r E_{n}^{2}(u, r-1)}\left[x_{1}^{\frac{1}{r}} \frac{\frac{1}{r}}{x_{2}^{r}}\left(x_{1}^{-1}-x_{2}^{-1}\right) A+\left(x_{1}^{\frac{1}{r}-1}-x_{2}^{\frac{1}{r}-1}\right) B\right] .
\end{aligned}
$$

It follows from (3.3) and Lemma 2.3 that

$$
\begin{aligned}
A & =\left(u_{1}+u_{2}\right)\left[E_{n-2}^{2}\left(u_{3}, \ldots, u_{n} ; r-2\right)-E_{n-2}\left(u_{3}, \ldots, u_{n} ; r-1\right)\right. \\
& \left.\times E_{n-2}\left(u_{3}, \ldots, u_{n} ; r-3\right)\right]+E_{n-2}\left(u_{3}, \ldots, u_{n} ; r-1\right) E_{n-2}\left(u_{3}, \ldots, u_{n} ; r-2\right) \\
& -E_{n-2}\left(u_{3}, \ldots, u_{n} ; r\right) E_{n-2}\left(u_{3}, \ldots, u_{n} ; r-3\right) \\
& >0 .
\end{aligned}
$$

Similarly, we can get $B>0$. 
It follows from the function $\frac{k-r}{x}(k=0,1)$ is decreasing in $(0,+\infty)$ that

$$
\left(x_{1}-x_{2}\right)\left(x_{1}^{\frac{k-r}{r}}-x_{2}^{\frac{k-r}{r}}\right) \leq 0,(k=0,1) \text {. }
$$

Therefore, inequality (3.1) follows from (3.6) and (3.7) together with $A>0$ and $B>0$. Next, we prove that $\phi_{r}(x)=\frac{F_{n}(x, r)}{F_{n}(x, r-1)}$ is increasing with respect to $x_{i}(i=1,2, \ldots, n)$.

By the symmetry of $\varphi_{r}(x)$ with respect to $x_{i}(i=1,2, \ldots, n)$, we only need to prove that

$$
\frac{\partial \phi_{r}(x)}{\partial x_{1}} \geq 0
$$

which can be derived directly from $A>0$ and $B>0$ together with Equation (3.4).

Proof of Theorem 1.2. It is obvious that $\phi_{r}^{*}(x)$ is symmetric and has continuous partial derivatives in $\mathbb{R}_{+}^{n}$. By Lemma 2.1, we only need to prove that

$$
\left(x_{1}-x_{2}\right)\left(\frac{\partial \phi_{r}^{*}(x)}{\partial x_{1}}-\frac{\partial \phi_{r}^{*}(x)}{\partial x_{2}}\right) \leq 0 .
$$

For any fixed $2 \leq r \leq n$, let $u_{i}=\sqrt[r]{x_{i}}, i=1,2, \ldots, n$ and $u=\left(u_{1}, u_{2}, \ldots, u_{n}\right) \in \mathbb{R}_{+}^{n}$. Then,

$$
\phi_{r}^{*}(x)=\frac{F_{n}^{*}(x, r)}{F_{n}^{*}(x, r-1)}=\frac{C_{r}(u)}{C_{r-1}(u)} .
$$

Differentiating $\phi_{r}^{*}(x)$ with respect to $x_{1}$, we have

$$
\frac{\partial \phi_{r}^{*}(x)}{\partial x_{1}}=\frac{1}{C_{r-1}^{2}(u)}\left[C_{r-1}(u) \frac{\partial C_{r}(u)}{\partial u_{1}} \frac{\partial u_{1}}{\partial x_{1}}-C_{r}(u) \frac{\partial C_{r-1}(u)}{\partial u_{1}} \frac{\partial u_{1}}{\partial x_{1}}\right] .
$$

It follows from Lemma 2.4 that

$$
\begin{aligned}
\frac{\partial C_{r}(u)}{\partial u_{1}} & =C_{r-1}(u)+u_{1} \frac{\partial C_{r-1}(u)}{\partial u_{1}} \\
& =C_{r-1}(u)+u_{1}\left[C_{r-2}(u)+u_{1} \frac{\partial C_{r-2}(u)}{\partial u_{1}}\right] \\
& =C_{r-1}(u)+u_{1} C_{r-2}(u)+u_{1}^{2} \frac{\partial C_{r-2}(u)}{\partial u_{1}} \\
& =\cdots \cdots \\
& =C_{r-1}(u)+u_{1} C_{r-2}(u)+u_{1}^{2} C_{r-3}(u)+\cdots+u_{1}^{r-2} C_{1}(u)+u_{1}^{r-1} .
\end{aligned}
$$

Equations (3.10) and (3.11) lead to

$$
\begin{aligned}
\frac{\partial \phi_{r}^{*}(x)}{\partial x_{1}} & =\frac{1}{C_{r-1}^{2}(u)}\left\{\left[C_{r-1}^{2}(u)-C_{r}(u) C_{r-2}(u)\right]+u_{1}\left[C_{r-1}(u) C_{r-2}(u)\right.\right. \\
& \left.-C_{r}(u) C_{r-3}(u)\right]+\cdots+u_{1}^{r-2}\left[C_{r-1}(u) C_{1}(u)-C_{r}(u) C_{0}(u)\right] \\
& \left.+C_{r-1}(u) u_{1}^{r-1}\right\} \frac{1}{r} u_{1}^{1-r} .
\end{aligned}
$$


Similarly, we have

$$
\begin{aligned}
\frac{\partial \phi_{r}^{*}(x)}{\partial x_{2}} & =\frac{1}{C_{r-1}^{2}(u)}\left\{\left[C_{r-1}^{2}(u)-C_{r}(u) C_{r-2}(u)\right]+u_{2}\left[C_{r-1}(u) C_{r-2}(u)\right.\right. \\
& \left.-C_{r}(u) C_{r-3}(u)\right]+\cdots+u_{2}^{r-2}\left[C_{r-1}(u) C_{1}(u)-C_{r}(u) C_{0}(u)\right] \\
& \left.+C_{r-1}(u) u_{2}^{r-1}\right\} \frac{1}{r} u_{2}^{1-r} .
\end{aligned}
$$

From (3.12) and (3.13), one has

$$
\begin{aligned}
& \left(x_{1}-x_{2}\right)\left(\frac{\partial \phi_{r}^{*}(x)}{\partial x_{1}}-\frac{\partial \phi_{r}^{*}(x)}{\partial x_{2}}\right) \\
& =\frac{x_{1}-x_{2}}{r C_{r-1}^{2}(u)}\left\{\left[C_{r-1}^{2}(u)-C_{r}(u) C_{r-2}(u)\right]\left(\frac{1-r}{x_{1}}-\frac{1-r}{r}\right)+\left[C_{r-1}(u) C_{r-2}(u)\right.\right. \\
& \left.-C_{r}(u) C_{r-3}(u)\right]\left(x_{1}^{\frac{2-r}{r}}-\frac{2-r}{r}\right)+\cdots+\left[C_{r-1}(u) C_{1}(u)-C_{r}(u) C_{0}(u)\right] \\
& \left.\times\left(x_{1} \frac{(r-1)-r}{r}-x_{2} \frac{(r-1)-r}{r}\right)\right\} .
\end{aligned}
$$

By Lemma 2.5, we know that

$$
\begin{aligned}
& C_{r-1}^{2}(u)-C_{r}(u) C_{r-2}(u)>0, \\
& C_{r-1}(u) C_{r-2}(u)-C_{r}(u) C_{r-3}(u)>0, \\
& \cdots \cdots, \\
& C_{r-1}(u) C_{1}(u)-C_{r}(u) C_{0}(u)>0 .
\end{aligned}
$$

The monotonicity of the function $\frac{j-r}{r}(1 \leq j \leq r-1)$ in $(0,+\infty)$ leads to the conclusion that

$$
\left(x_{1}-x_{2}\right)\left(x_{1}^{\frac{j-r}{r}}-x_{2}^{\frac{j-r}{r}}\right) \leq 0 .
$$

Therefore, inequality (3.8) follows from (3.14)-(3.16).

Next, we prove that $\phi_{r}^{*}(x)=\frac{F_{n}^{*}(x, r)}{F_{n}^{*}(x, r-1)}$ is increasing with respect to $x_{i}(i=1,2, \ldots, n)$.

From (3.12) and (3.15), we clearly see that

$$
\frac{\partial \phi_{r}^{*}(x)}{\partial x_{1}} \geq 0 .
$$

Inequality (3.17) implies that $\phi_{r}^{*}(x)$ is increasing with respect to $x_{1}$, then from the symmetry of $\phi_{r}^{*}(x)$ with respect to $x_{i}(i=1,2, \ldots, n)$ we know that $\phi_{r}^{*}(x)$ is increasing with respect to each $x_{i}(i=1,2, \ldots, n)$.

Proof of Corollary 1.1. By Theorem 1.1 and Lemma 2.6, we have $\phi_{r}\left(\frac{c-x}{\frac{n c}{s}-1}\right) \geq \phi_{r}(x)$ and $\phi_{r}\left(\frac{c+x}{s+n c}\right) \geq \phi_{r}\left(\frac{x}{s}\right)$ which imply Corollary 1.1. 
Remark 1. Let $0<x_{i} \leq \frac{1}{2}, i=1,2, \ldots, n$, then

$$
\frac{G_{n}(x)}{G_{n}(1-x)} \leq \frac{A_{n}(x)}{A_{n}(1-x)}
$$

where $(1-x)=\left(1-x_{1}, 1-x_{2}, \ldots, 1-x_{n}\right)$, commonly referred to as Ky Fan inequality (see [15]), which has attracted the attention of a considerable number of mathematicians (see [16-20]).

Letting $\sum_{i=1}^{n} x_{i} \leq 1$ and taking $c=1$ in Corollary 1.1, we get

$$
\frac{G_{n}(x)}{G_{n}(1-x)}=\frac{F_{n}(x, n)}{F_{n}(1-x, n)} \leq \frac{F_{n}(x, n-1)}{F_{n}(1-x, n-1)} \leq \cdots \leq \frac{F_{n}(x, 1)}{F_{n}(1-x, 1)}=\frac{A_{n}(x)}{A_{n}(1-x)} .
$$

It is obvious that inequality (3.19) can be called Ky Fan-type inequality.

Remark 2. Let $x_{i}>0, i=1,2, \ldots, n$, the following inequalities

$$
\prod_{i=1}^{n}\left(x_{i}^{-1}-1\right) \geq(n-1)^{n}
$$

and

$$
\prod_{i=1}^{n}\left(x_{i}^{-1}+1\right) \geq(n+1)^{n}
$$

are the well-known Weierstrass inequalities (see [11]).

Taking $c=s=1$ in Corollary 1.1, one has

$$
\prod_{i=1}^{n}\left(x_{i}^{-1}-1\right) \geq\left(\frac{F_{n}(1-x, n-1)}{F_{n}(x, n-1)}\right)^{n} \geq \cdots \geq\left(\frac{F_{n}(1-x, 2)}{F_{n}(x, 2)}\right)^{n} \geq(n-1)^{n}
$$

and

$$
\prod_{i=1}^{n}\left(x_{i}^{-1}+1\right) \geq\left(\frac{F_{n}(1+x, n-1)}{F_{n}(x, n-1)}\right)^{n} \geq \cdots \geq\left(\frac{F_{n}(1+x, 2)}{F_{n}(x, 2)}\right)^{n} \geq(n+1)^{n} .
$$

It is obvious that our inequalities can be called Weierstrass-type inequalities.

Proof of Corollary 1.2. By Theorem 1.2 and Lemma 2.6, we have $\phi_{r}^{*}\left(\frac{c-x}{\frac{n c}{s}-1}\right) \geq \phi_{r}^{*}(x)$ and $\phi_{r}^{*}\left(\frac{c+x}{s+n c}\right) \geq \phi_{r}^{*}\left(\frac{x}{s}\right)$, which imply Corollary 1.2.

\section{Acknowledgements}

This work was supported by NSF of China under grant No. 11071069.

\section{Competing interests}

The author declares that he has no competing interests.

Received: 22 February 2011 Accepted: 5 December 2011 Published: 5 December 2011

\section{References}

1. Hara, T, Uchiyama, M, Takahasi, S: A refinement of various mean in-equalities. J Inequal Appl. 2(4), $387-395$ (1998) Guan, KZ: The Hamy symmetric function and its generalization. Math Inequal Appl. 9(4), 797-805 (2006)

Marshall, AW, Olkin, I: Inequalities: Theorey of Majorization and Its Applications. Academic Press, New York (1979)

. Hwang, FK, Rothblum, UG: Partition-optimization with Schur convex sum objective functions. SIAM J Discret Math. 18, 512-524 (2004). doi:10.1137/S0895480198347167 
5. Zhang, XM: Schur-convex functions and isoperimetric inequalities. Proc Am Math Soc. 126(2), 461-470 (1998). doi:10.1090/S0002-9939-98-04151-3

6. Stepniak, C: Stochastic ordering and Schur-convex functions in comparison of linear experiments. Metrika. 36(5), 291-298 (1989)

7. Constantine, GM: Schur convex functions on spectra of graphs. Discret Math. 45(2-3), 181-188 (1983). doi:10.1016/0012365X(83)90034-1

8. Merkle, M: Convexity, Schur-convexity and bounds for the gamma function involving the digamma function. Rocky Mountain J Math. 28(3), 1053-1066 (1998). doi:10.1216/rmjm/1181071755

9. Hwang, FK, Rothblum, UG, Shepp, L: Monotone optimal multipartitions using Schur convexity with respect to partial orders. SIAM J Discret Math. 6(4), 533-547 (1993). doi:10.1137/0406042

10. Chan, NN: Schur-convexity for A-optimal designs. J Math Anal Appl. 122(1), 1-6 (1987). doi:10.1016/0022-247X(87)903398

11. Bullen, PS: Handbook Of Means And Their Inequalities. Kluwer Academic Publishers Group, Dordrecht (2003)

12. Guan, KZ: Schur-convexity of the complete symmetric function. Math Inequal Appl. 9(4), 567-576 (2006)

13. Menon, KV: Inequalities for symmetric functions. Duke Math J. 35, 37-45 (1968). doi:10.1215/50012-7094-68-03504-7

14. Shi, HN: Refinement and generalization of a class of inequalities for symmetric functions. (Chinese) Math Pract Theory. 29(4), $81-84$ (1999)

15. Beckenbach, EF, Bellman, R: Inequalities. Springer, New York (1965)

16. Alzer, H: On an inequality of Ky Fan. J Math Anal Appl. 137(1), 168-172 (1989). doi:10.1016/0022-247X(89)90280-1

17. Alzer, H: The inequality of Ky Fan and related results. Acta Appl Math. 38(3), 305-354 (1995). doi:10.1007/BF00996150

18. Neuman, E, Sándor, J: On the Ky Fan inequality and related inequalities. II Bull Aust Math Soc. 72(1), 87-107 (2005). doi:10.1017/50004972700034894

19. Govedarica, V, Jovanović, M: On the inequalities of Ky Fan, Wang-Wang and Alzer. J Math Anal Appl. 270(2), 709-712 (2002). doi:10.1016/S0022-247X(02)00074-4

20. Dragomir, SS, Scarmozzino, FP: On the Ky Fan inequality. J Math Anal Appl. 269(1), 129-136 (2002). doi:10.1016/S0022$247 \times(02) 00009-4$

doi:10.1186/1029-242X-2011-131

Cite this article as: Qian: Schur convexity for the ratios of the Hamy and generalized Hamy symmetric functions. Journal of Inequalities and Applications 2011 2011:131.

\section{Submit your manuscript to a SpringerOpen ${ }^{\odot}$} journal and benefit from:

Convenient online submission

Rigorous peer review

- Immediate publication on acceptance

- Open access: articles freely available online

- High visibility within the field

- Retaining the copyright to your article

Submit your next manuscript at $\boldsymbol{s p r i n g e r o p e n . c o m ~}$ 